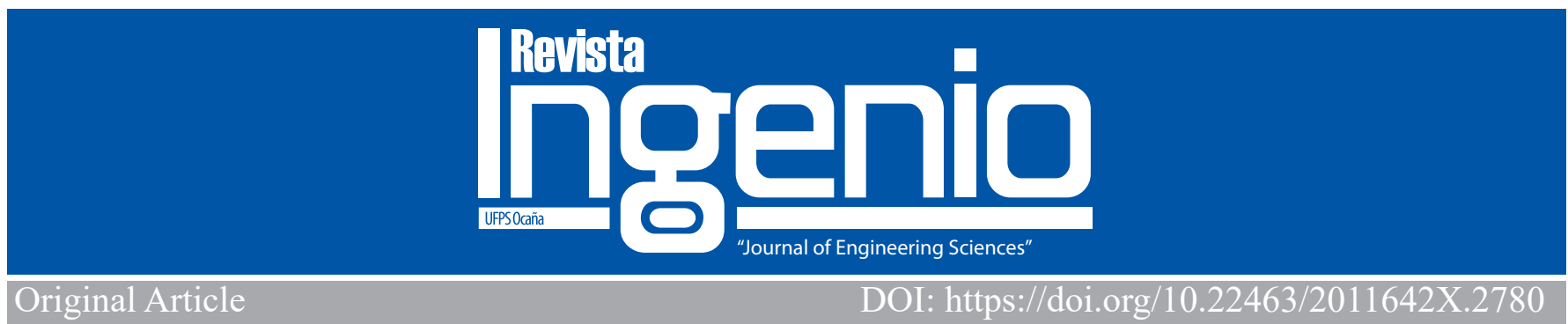

\title{
Sistema de gestión de energía bajo el paradigma de Industria 4.0
}

Energy management system under the Industry 4.0 paradigm

Ing. Julián Uribe-Hernández ${ }^{1}$, Ing. Laura Avila-Roa', Ph.D Edgar Alfonso Chacón-Ramirez²

SIMAC-S.A., Medellin-Colombia, https://orcid.org/0000-0002-1309-0856, julian.uribe@simac.com.co, https://orcid.org/0000-0001-5199-4082 laura.avila@simac.com.co

Escuela de Ingeniería de Sistemas, Facultad de Ingeniería, Universidad de Los Andes, Mérida-Venezuela, Orcid: https://orcid.org/0000-0001-5612-0368, echacon@ula.ve

Como citar: J. Uribe, L. Avila \& E. A. Chacón, "Ssitema de gestión de energía bajo el paradigma de Industria 4.0”, Revista Ingenio, 18(1), pp. 33-40, 2021

\section{RESUMEN}

Palabras claves:

Automatización de Procesos de Negocio, Industria 4.0, ISO 50001, PyMEs
El estándar ISO 50001 para la gestión de la energía es bien conocido en el contexto de las Pequeñas y Medianas Empresas, aunque su aplicación no es común. Por otra parte, ha aparecido en esta década un paradigma que es el de Industria 4.0 (I 4.0) donde la gestión eficiente de la energía es uno de los elementos centrales. Ambos conceptos son de gran importancia para la mejora de las PyMEs, pero éstas no cuentan con las herramientas que permitan ir incorporando estos conceptos a su entorno. En el trabajo se muestra como se adquieren la información de los tres ejes del modelo Rami 4.0 mediante la incorporación de una herramienta para la gestión de energía que permitirá a los usuarios mejorar la eficiencia energética. Resultados de la aplicación del sistema son mostrados.

\section{ABSTRACT}

Keywords:

Robotic Process

Automation ,Industry 4.0,

ISO 50001, SMEs
The ISO 50001 standard for energy management is well known in the context of Small and Medium Enterprises, although its application is not common. On the other hand, a paradigm has appeared in this decade, namely Industry 4.0 (I 4.0) where efficient energy management is one of the central elements. Both concepts are of great importance for the improvement of SMEs, but they do not have the tools that allow them to incorporate these concepts into their environment. The work shows how the information on the three axes of the Rami 4.0 model is acquired through the incorporation of an energy management tool that will allow users to improve energy efficiency. Results of the system application are shown.

\section{Introducción}

La industria 4.0 (I4.0) es un concepto relativamente nuevo asociado a la búsqueda de la eficiencia en la industria y que se caracteriza por tener: a) Cortos períodos en la incorporación de un nuevo producto; b) Personalización de la demanda; c) Flexibilidad, para responder de manera rápida a la demanda del consumidor; d) Descentralización, para mayor agilidad y e) Eficiencia en el manejo de los recursos, que está asociado a costos, cambio social y a los aspectos ecológicos [1]. En las empresas que siguen este enfoque, sus procesos están fuertemente integrados tanto horizontalmente por el Ciclo de vida del producto y la cadena de valor, y verticalmente por la interacción entre los procesos de producción y los procesos de gestión del negocio mediante el uso de Tecnologías de Información y Tecnologías de Operación [2]. Las interacciones entre procesos de producción y procesos del negocio se dan por la incorporación del conocimiento formal en todos sus procesos de toma de decisiones, mediante la digitalización de procedimientos, información y modelos de comportamiento de recursos y procesos, lo que permite tener una imagen completa de los distintos procesos de producción lo que permite la toma de decisiones de manera automática o semiautomática. La eficiencia de la empresa incluye la satisfacción del cliente y la sostenibilidad de la empresa. La sostenibilidad de la industria está asociado a la eficiencia de los recursos y a la eficiencia energética [3] donde la eficiencia energética afecta tanto en la competitividad económica [4-5] como el cumplimiento del compromiso social de tener empresas limpias (de producción verde) [4-6].

Las características de estas nuevas industrias están asociadas al uso de los conceptos de Cyber Physical Systems (CPS), Cyber Physical Production Systems (CPPS), "Cloud and Fog computing", y "big data analytics" todas estas orientadas a lograr tener una 
producción inteligente [7]. Para la implantación de I 4.0, se ha propuesto una Arquitectura de Referencia denominada "Reference architectural model industrie 4.0" (Rami 4.0) [8] y que se muestra en la Figura 1.

LaArquitectura de referencia presenta 3 dimensiones que son: 1) la del ciclo de vida y cadena de valor, que cubre la integración horizontal, 2) la dimensión asociada a los niveles de jerarquía de los activos de producción integración vertical de los activos y que sigue la propuesta de la ISA; y 3) la dimensión asociada a las capas funcionales necesarias para la gestión y el conocimiento de procesos, productos, estado de la planta. Este modelo exige tener una información completa de los modelos de procesos, productos, jerarquía de la planta, relaciones entre procesos, instrumentación en el piso de planta, que no siempre es conocida por las PyMEs [9-10-11-12-14]. La gestión de la energía es una de las funciones fundamentales en el enfoque I 4.0, y esta gestión toma en cuenta rutas de productos (cadena de valor), modelos de productos y procesos, relacionados con la dimensión del producto; puntos de alimentación de energía, sensores, asociados a la jerarquía de los equipos y el flujo de los datos e información, relacionada con la dimensión de las funciones y la infraestructura tecnológica que es la otra dimensión del modelo Rami 4.0. Desde el punto de vista de sistemas de Gestión de Energía (EMS), el proceso de implantación de este tipo de sistemas es muy bajo por distintas razones, entre las cuales están el costo de implantar equipos de medición y el desconocimiento como utilizar los resultados del análisis y de donde ubicar el sistema de gestión de energía, pues no poseen un Sistema de Gestión de Operaciones (MES) [15].

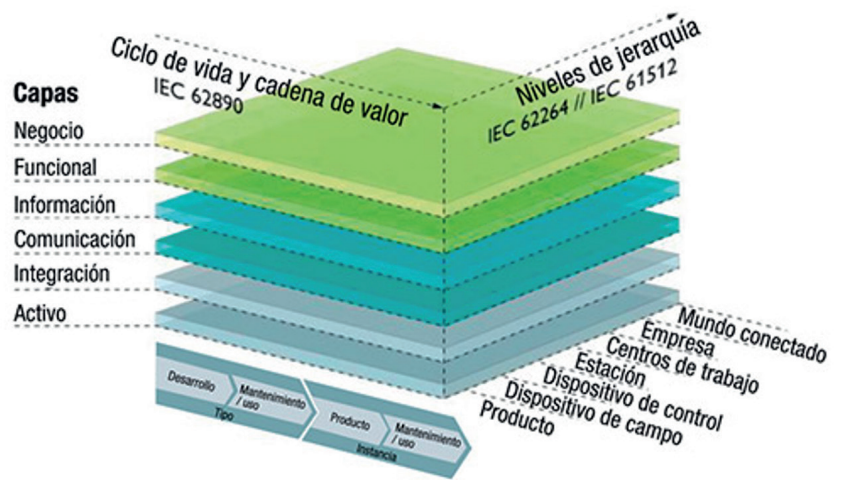

Figura 1. Arquitectura de Referencia para Industria 4.0. Fuente [9].

Las PyMEs necesitan sistemas de apoyo a la gestión de sus procesos dentro del marco de I 4.0. Estas herramientas deben ser capaces de "extraer información" que permita incorporar el conocimiento de sus procesos, estructura y completar los modelos para realizar la gestión integral del proceso de gestión de energía.

El objetivo del presente trabajo es mostrar como en la implantación de un sistema de gestión de energía según la norma ISO 50001, y al cual se le agregan mecanismos de captura de información acorde a Rami 4.0, se obtienen resultados que permiten una mejora del rendimiento en el proceso de producción y se introduce el concepto de integración asociado a I 4.0.

El trabajo está organizado de la siguiente manera, una introducción donde se destaca la necesidad de sistemas de gestión de energía acordes con el paradigma I 4.0. La sección 2 presenta de manera breve la norma ISO 50001 y sus necesidades de información y como ajustarla a I 4.0. La sección 3 presenta la metodología empleada para describir el proceso de producción, La sección 4 la arquitectura funcional del sistema para cumplir con los requerimientos de I 4.0 e ISO 50001. Los resultados de la implantación se dan en la sección 5 , y finalmente se dan las conclusiones.

\section{ISO 50001 para I 4.0}

2.1. Requerimientos para un sistema de gestión de energía. El cambio climático ha generado que las naciones del mundo tiendan a establecer políticas que tiendan a una reducción de las emisiones de carbono para reducir el efecto invernadero, por una parte, y por otra el costo de los combustibles hace que se trate de reducir el consumo de los mismos mediante una mejora en la eficiencia de los procesos industriales. El Organismo Internacional de Estándares (ISO) propuso una metodología de mejora continua conocida como ISO 50001 derivada de ISO 9001 e ISO 14001 teniendo como objetivo la introducción de modificaciones en recursos, procedimientos, hábitos que mejoren el rendimiento energético en la industria [16-19]. Una lista de los requerimientos que deben tener los sistemas de gestión de ISO 50001, tal como se muestra en [16-17], para ser compatibles con I 4.0 se dan a continuación:

1. El sistema debe estar localizado en el sitio de uso

2. El acceso a la información debe ser vía intranet.

3. Una lista de las acciones posibles de mejora deben estar documentadas y disponibles en el sistema.

4. La gestión documental debe ser parte del sistema.

5. La estructura de los sensores (mediciones) debe ser posible dentro de la jerarquía del equipamiento

6. Las medidas de los sensores deben estar disponibles gráficamente.

7. Valores límites para las medidas son editables, y la violación de esos límites deben generar 
alarmas.

8. Los indicadores de eficiencia pueden ser definidos y ser visualizados gráficamente

9. Debe existir un generador de reportes.

2.2. El ciclo del proceso de mejora continua en ISO 50001 La norma ISO 50001 se basa en el concepto de mejora continua que consta de tres pasos principales: a) Planificar, b) Ejecutar, c) Verificar y d) Actuar, (PDCA por sus siglas en inglés). Una breve descripción del ciclo de gestión energética según la norma ISO 50001, ver Figura 2, se da a continuación.

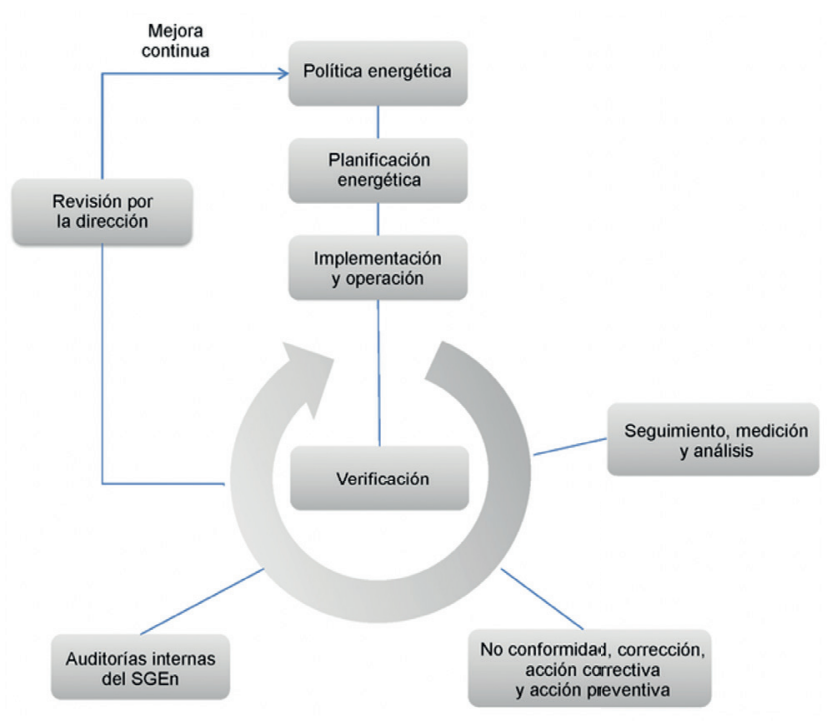

Figura 2. Modelo de Gestión de Energía Fuente [13]

Planificación. Consiste en el establecimiento de la política energética para la organización fijando los objetivos de consumo de energía o los indicadores apropiados para la organización. En este paso se establecen los usos significativos de la energía, estableciendo los principales puntos donde se debe actuar para reducir los consumos y/o mejorar la eficiencia. Finalmente, se establece un plan para el siguiente período especificando un conjunto de actividades a ser ejecutadas.

Ejecución. Consiste en la implementación de los planes establecidos para un período, mediante la ejecución de las actividades programadas en el paso anterior. Las distintas actividades ejecutadas serán registradas y se inicia un seguimiento para verificar el impacto que ha tenido la ejecución del plan.

Verificación. En este paso se realiza un seguimiento de las operaciones de la organización para determinar cómo ha cambiado el consumo o mejorado el rendimiento de los procesos. En el caso de que el impacto no haya sido el esperado se comprueba si la ejecución de las actividades ha sido bien realizada.

Actuar. En este paso se determina el impacto de la ejecución del plan en el consumo energético y se determinan las posibles fallas en la planificación que condijeron a obtener resultados distintos a los esperados.

El establecimiento de los casos de "uso significativo de consumo" y los indicadores para la empresa, implica en la fase de planificación tomar en consideración las dimensiones del modelo Rami 4.0 mostrado en la Figura 1. Por lo tanto, la selección de los puntos de medición, frecuencia de mediciones, estrategia de cálculo para los indicadores, bajo el enfoque de I 4.0 implica tomar en cuenta la cadena de valor para cada producto, y el consumo de energía para el mismo en las distintas órdenes de producción. Las mediciones y los indicadores seleccionados deben permitir calcular índices para cada dimensión y las funciones de cada capa. Las mediciones de rendimiento de energía en I 4.0 está asociado al concepto de "energía embebida" en el producto, que implica que toman en cuenta las distintas actividades internas en la empresa y la cantidad de energía que ya poseen las materias primas [20-21-22].

\section{Modelado del proceso de producción incluyendo energía.}

Un proceso de producción consiste en la transformación de unos materiales de entrada en un nuevo producto, o un producto mejorado mediante el uso de recursos humanos, equipos y un consumo de energía tal como se muestra en la Figura 3.

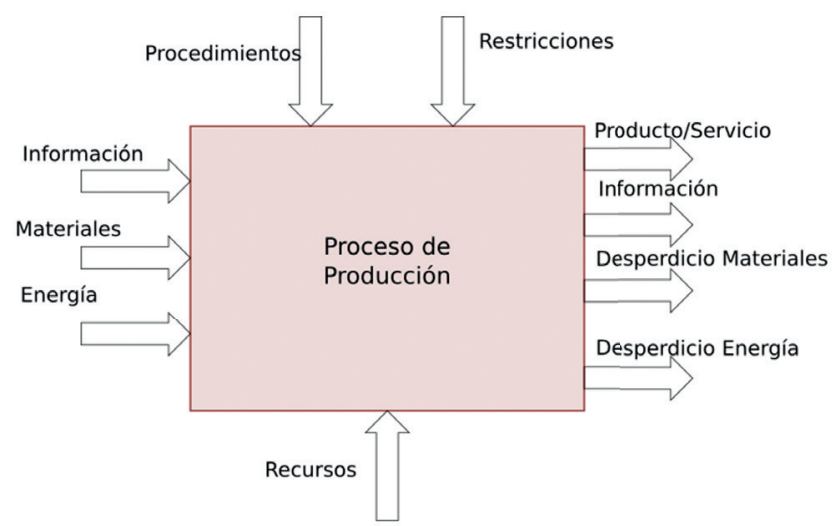

Figura 3. Proceso de Producción. Fuente: Modificado de [17] 
En [18] se proponen algunos indicadores y la arquitectura de automatización asociada a I 4.0 para mejorar la eficiencia energética, mediante la utilización de indicadores en la fase de programación de la producción. Los indicadores propuestos para el sistema desarrollado bajo este concepto el sistema son:

- Eficiencia de equipo con relación a un consumo teórico del equipo.

- Eficiencia de un área de producción con respecto a un consumo teórico esperado para un lapso de tiempo.

- Eficiencia para la obtención de un producto en una ruta.

- Eficiencia de una unidad de producción para una etapa del producto.

La energía está asociada al recurso físico -Dimensión lateral en Rami 4.0 y jerarquía de equipamiento en ISA 95 - para poder efectuar sus funciones. Los sensores de energía se ubican físicamente en relación a esa dimensión. Con respecto a la dimensión vertical, cubre desde el activo, donde está la interfaz de lo físico con lo digital, hasta la capa de las funciones del negocio, el sensor adquiere la información, y está será transmitida hasta la capa información de esta dimensión donde están las imágenes (gemelos digitales) de procesos y recursos [23].

Las mediciones de energía, como las otras mediciones deben permitir determinar el cumplimiento de las distintas funciones asociadas a la gestión de las operaciones de producción, y determina la ubicación de los sensores de energía que permitan conocer el consumo y rendimiento por producto y sus rutas, así como el consumo por equipos y la jerarquía de activos y que van a permitir el apoyo a la toma de decisiones tanto en los niveles de gestión de la producción como en los procesos del negocio.

La fase de planificación debe suministrar la información sobre la jerarquía de activos, "Rutas de Producto" y "Modelos de Producto" para poder establecer los indicadores asociados a esta dimensión, o el sistema de seguimiento debe tener la inteligencia para ir construyendo los modelos, y de esa manera generar los indicadores asociados al "Modelo del Producto" y "Rutas de Producto". En las empresas visitadas, no existe la información completa sobre Modelo de Producto, ni la conectividad de los recursos que les permita definir de manera automática las mejores rutas para un producto. El sistema debe aprender las rutas y modelos de producto a partir de la captura de información en el seguimiento.
La eficiencia energética está asociada al consumo de energía en el proceso con relación al producto o servicio obtenido en el proceso. El objetivo es el de reducir el consumo de energía y/o aumentar la cantidad de Producto / Servicio. Las pérdidas de energía están asociadas al desperdicio por la cantidad de energía que se desprende como calor por falta de mantenimiento en los equipos, bajo aislamiento en hornos, baja calidad en el proceso lo que conlleva pérdida de materiales. Los indicadores de eficiencia deben tomar en cuenta esos factores que influyen en el consumo de energía y en la eficiencia de la producción.

El sistema tiene como fuentes de alimentación de información las mediciones de energía, los datos de la jerarquía de equipamiento y los datos de las órdenes de producción. La información de la jerarquía de equipamiento y los puntos de medición de energía (sensores) son introducidos manualmente cuando se definen los "casos de uso significativo", el sistema de medición de energía captura la información de los sensores registrando el consumo periódicamente con una frecuencia del minuto. La información de las órdenes de producción se recuperan de los registros que se llevan para cada orden donde se registra la cantidad de producto, los equipos que se utilizan en esa orden y la fecha de inicio, fin de actividad de cada equipo para una orden.

3.1 Esquema de aprendizaje para de los modelos de producto y rutas de producto.

La información de Modelos de Producto y Rutas de producto no están disponibles en muchas PyMEs, por lo cual se implementó el método que se describe a continuación:

- Cada orden se analiza y se verifica si para ese producto ya existe una orden anterior, o en el caso de que el producto no haya sido registrado, se crea un nuevo producto.

- Para cada producto, se determina si los equipos utilizados ya han sido registrados con anterioridad, o si no existen se crea una nueva ruta para el producto.

- Se registra la información de la orden para ser analizada posteriormente

- Se calcula el índice y se compara con los indicadores asociados a producto, ruta, equipamiento. 


\section{Descripción de Enerbot}

4.1 Sistema local vía intranet

El sistema de gestión energética "Enerbot" cumple con los requerimientos dados en la sección 2 para un sistema de gestión de energía compatible con I 4.0 y que han sido modificados de [16]. Es un sistema que presenta un acceso vía web en la intranet de la empresa. En la figura 4, se muestra la arquitectura de equipos y redes utilizada para implantar el sistema, manteniendo la arquitectura presente en la mayoría de instalaciones de automatización.

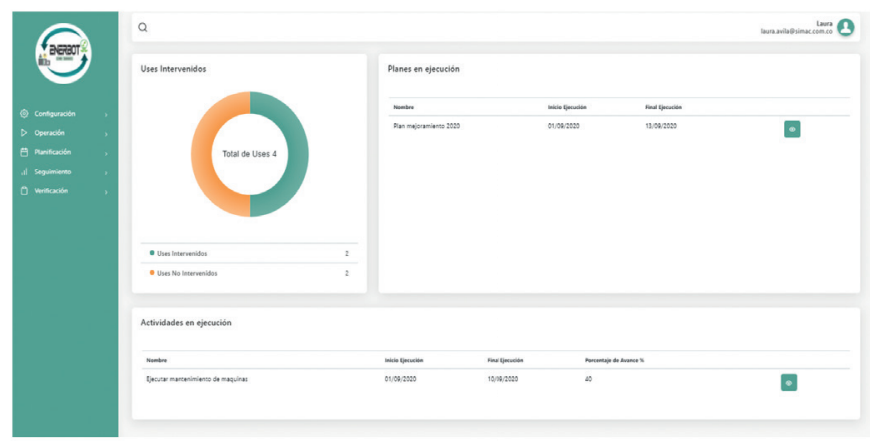

Figura 4. Enerbot página de inicio.

La aplicación fue construida en PHP usando el "framework Laravel" de acuerdo a las especificaciones del grupo de desarrollo para toda la interacción con los usuarios. Un simulador construido en python para el cálculo de los modelos de línea base para los indicadores.

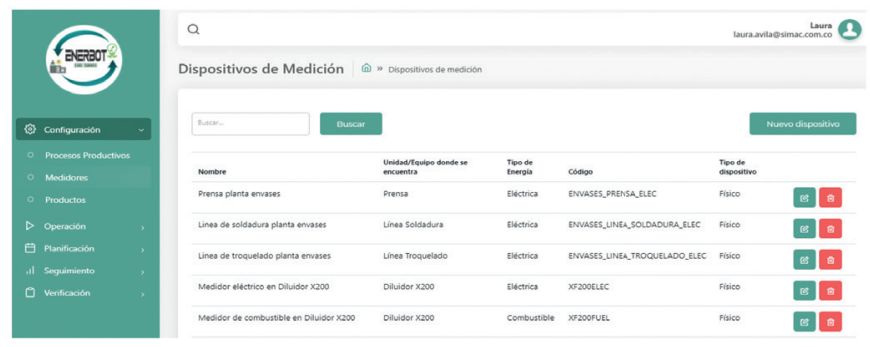

Figura 5. Pantalla de especificación de sensores de medición.

La navegación en el sistema sigue el esquema de ISO 50001, tal como se muestra en la Figura 5, pero existe una fase adicional que permite la especificación de la arquitectura de la planta y los procesos que se desarrollan en las distintas unidades con los cuales se define la ubicación de los distintos sensores de medición de energía.

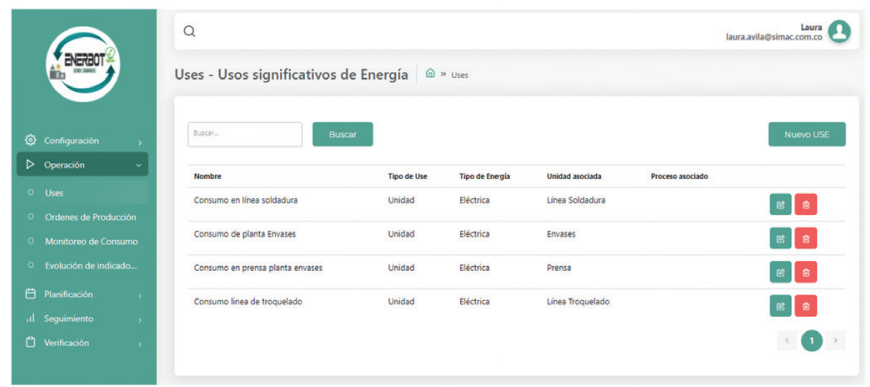

Figura 6. Definición de los caso de uso significativo.

En la fase de planificación, se crean los "Use Cases" mediante la interfaz mostrada en la Figura 7.

En la fase de verificación, el sistema adquiere los datos mediante la captura de las órdenes de producción que son generados por el sistema de planificación de la empresa y que se muestran en la Figura 8.

\subsection{Integración con el sistema de medición de energía}

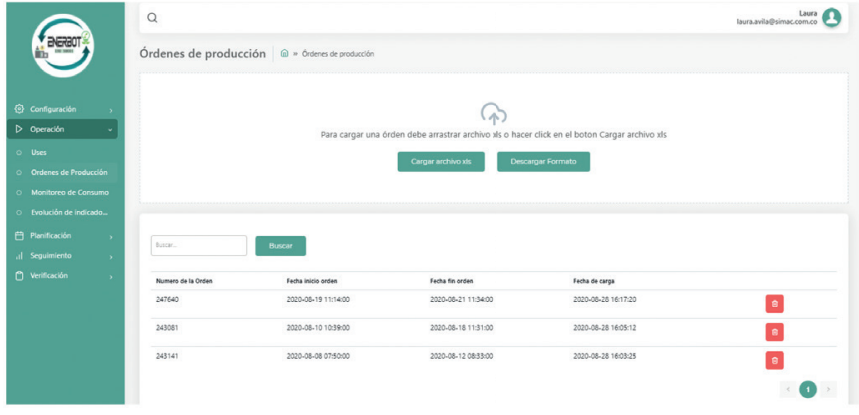

Figura 7. Captura de la información de las órdenes de producción.

El sistema de medición de energía utilizado se construyó para la captura de información del consumo energético usando tecnología dispositivos para IoT (Ver Figura 4). Los datos son recolectados y almacenados en un historiador que mantiene las medidas de los distintos sensores con contadores de consumo eléctrico, donde se registra el valor del medidor y la fecha. Para establecer las relaciones de consumo con las órdenes de producción, el sistema recupera las fechas de las actividades de cada orden y las une con los datos del historiador de energía.

\subsection{Despliegues del sistema}

Los datos recolectados por el sistema son almacenados y se asocian a las distintas órdenes de producción. Se pueden tener datos por máquina, por proceso y por orden de producción tal como se muestra en las figuras 8 y 9 . 


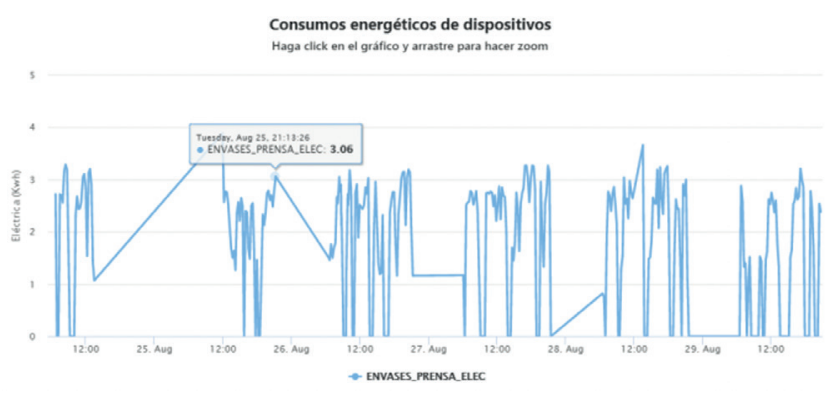

Figura 8. Registros de consumo del equipo prensa hidráulica.

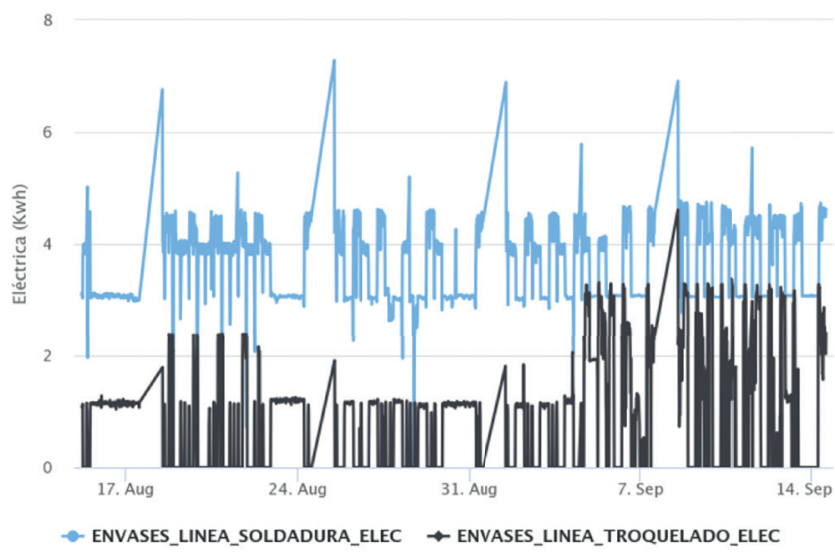

Figura 9. Despliegues de consumo por líneas de producción.

\section{Análisis de los resultados}

5.1. Descripción de la jerarquía de equipos, ubicación de sensores asociados a los Casos de Uso.

La introducción del sistema necesitó el acompañamiento de los especialistas para aclarar conceptos de industria 4.0 y de justificación sobre los puntos de medición y del porqué de los indicadores. Existe la idea de "consumos estándar" como un elemento de evaluación y no la idea de asociarlo al producto y las rutas de producto.

La opinión generalizada en las PyMEs visitadas, es medir el equipo independientemente de las rutas y los productos. Existe un desconocimiento de los conceptos de modelo de producto, pero el sistema trata de hacerlo transparente construyendo los internamente los modelos. El impacto de la herramienta con los resultados aún no se conoce por el poco tiempo de uso. El concepto de Gemelo Digital y como mantener la información de consumo en el gemelo es difícil de aceptar por lo que se habló de historia del recurso y del proceso.

Se creó conciencia acerca de la importancia de tener indicadores que tengan la relación de energía respecto a la programación de producción, y no solo respecto al equipo y su tiempo de uso.

\subsection{Usabilidad del sistema}

El sistema está en su uso inicial en una empresa de la región Antioqueña, para la validación experimental del mismo. Se procedió al entrenamiento del personal en la planta, donde el personal especialista en energía definió la política energética de la empresa, se seleccionaron los casos de uso y estableció un conjunto de actividades a ser desarrolladas para la mejora del rendimiento energético. El sistema ha mostrado su facilidad de uso y la integración con los sistemas de medición de energía y el sistema de manejo de las órdenes de producción.

El sistema facilita la integración de distintas fuentes de datos y automatiza el proceso de recolección e integración de la información, y de esta manera hace transparentea los usuarios los procedimientos repetitivos de búsqueda de la información, acondicionamiento, ajusto y preparación de reportes mensuales y de cierre de los ciclos de planificación de mejoramiento energético trabajando como un Robotic Process Automation.

\section{Conclusiones}

El consumo de energía afecta de distintas maneras a las empresas manufactureras, siendo su costo un factor fundamental en el precio de los productos por una parte y el otro elemento fundamental es la sostenibilidad desde el punto de vista de una producción "verde". En los últimos años de la década actual, el concepto de la cuarta revolución industrial se ha introducido fuertemente en la sociedad, y el factor del consumo energético forma parte de este concepto. Para las PyMEs, la introducción de sistemas de gestión de energía debe estar alineada con el concepto de Industria 4.0, para de esa manera ir incorporando estos conceptos de una manera suave, sin causar traumas.

El sistema desarrollado facilita la introducción de los conceptos de eficiencia energética y los formalismos de Industria 4.0, por lo que constituye una vía de alcanzar la transformación digital de una manera fácil para la organización, cumpliéndose los objetivos de lograr integrar la gestión energética asociado con los conceptos de I 4.0, utilizando la infraestructura de operaciones disponible.

\section{Agradecimiento}

Este trabajo contó con el apoyo del Ministerio de Ciencia y Tecnología de Colombia: "ENERBOT Energy and Production Analyst". Convocatoria "Cierre 
de Brechas Tecnológicas 2018”. El trabajo contó con el apoyo de la empresa de desarrollo de Software Active One en la implementación de las ideas aquí expuestas.

\section{Referencias}

[1] Lasi, H., Fettke, P., Kemper, H. G., Feld, T., \& Hoffmann, M. (2014). Industry 4.0. Business \& information systems engineering, 6(4), 239-242. Doi: 1007/s12599-014-0334-4

[2] Stock, T., \& Seliger, G. (2016). Opportuni-ties of sustainable manufacturing in indus-try 4.0. Procedia Cirp, 40, 536-541. Doi: 10.1016/j.procir.2016.01.129

[3] Bartodziej, C. J. (2017). The concept indus-try 4.0. In The concept industry 4.0 (pp. 27-50). Springer Gabler, Wiesbaden. Doi: 10.1007/9783-658-16502-4 3

[4] Kamble, S. S., Gunasekaran, A., \& Gawankar, S. A. (2018). Sustainable Indus-try 4.0 framework: A systematic literature review identifying the current trends and fu-ture perspectives. Process Safety and Envi-ronmental Protection, 117, 408425. Doi: 10.1016/j.psep.2018.05.009

[5] Mohamed, N., Al-Jaroodi, J., \& Lazarova-Molnar, S. (2019). Leveraging the capabili-ties of industry 4.0 for improving energy efficiency in smart factories. Ieee Access, 7, 18008-18020. Doi: 0.1109/ACCESS.2019.2897045

[6] Carvalho, N., Chaim, O., Cazarini, E., \& Gerolamo, M. (2018). Manufacturing in the fourth industrial revolution: A positive pro-spect in sustainable manufacturing. Proce-dia Manufacturing, 21, 671-678. Doi: 10.1016/j.promfg.2018.02.170

[7] Trentesaux, D., Borangiu, T., \& Thomas, A. (2016). Emerging ICT concepts for smart, safe and sustainable industrial systems. Computers in Industry, 81, 1-10. Doi: 10.1016/j. compind.2016.05.001

[8] Schweichhart, K. (2016). Reference archi-tectural model industrie 4.0 (rami 4.0). An Introduction. Available online: https://www. plattform-i40. de I, 40.

[9] Revista Electro Industria. ARQUITECTURAS DE REFERENCIA RAMI 4.0 E IIRA Una cuestión de perspectiva y enfoquehttp:// www.emb.cl/electroindustria/articulo.mvc? X$\mathrm{id}=2856 \&$ ni=arquitecturas-de-referencia-rami-40-e-iira-una-cuestion-de-perspectiva-y-enfoque

[10] Glass, R., Meissner, A., Gebauer, C., Stürmer, S. \& Metternich, J. "Identifying the barriers to Industrie 4.0", Procedia CIRP, vol. 72, pp 985 $-988,2018$.

[11] Mittal, S., Khan, M. A., Romero, D., \& Wuest,
T. "A critical review of smart manu-facturing \& Industry 4.0 maturity models: Implications for small and medium-sized enterprises (SMEs)", Journal of Manufac-turing Systems, vol. 49, pp $194-214,2018$

[12] E. A. Chacón, J. J. Cardillo \& J. Uribe, "Industria 4.0 en América Latina: Una ruta para su implantación”, Revista Ingenio, 17 (1), pp.28-35, 2020. Doi: $10.22463 / 2011642 X .2386$

[13] Thimm, H. (2018). Energy Data Managment in an Eco Learning Factory with Traditional SME Characteristics. In From Science to Society. 95105. Springer, Cham Doi; 10.1007/978-3-31965687-8 9.

[14] Yacob, P., Maludin, N., Aziz, N. S., Nodesan, S., Zin, A. W. M., \& Keong, L. E. (2018). Acceptability of sustainable green practices: perception of electrical \& elec-tronics manufacturing SMEs. Chemical En-gineering Transactions, 63, 319324. Doi: 10.3303/CET1863054

[15] Fernando, Y., \& Hor, W. L. (2017). Im-pacts of energy management practices on energy efficiency and carbon emissions re-duction: a survey of Malaysian manufactur-ing firms. Resources, Conservation and Re-cycling, 126, 62-73. Doi: 10.1016/j.resconrec.2017.07.023

[16] Thorsten Fiedler and Paul-Mihai Mircea. Energy management 130 systems according to the iso 50001 standard - challenges and benefits. In 2012 International Conference on Applied and Theoretical Electricity (ICATE), pages 1-4. IEEE, 2012. Doi: 0.1109/ICATE.2012.6403411

[17] Javied, T., Huprich, S., \& Franke. J. Cloud based energy management system compati-ble with the industry 4.0 requirements. IFAC-PapersOnLine, 52(10):171 - 175, 2019. 13th IFAC Workshop on Intelligent Manufacturing Systems IMS 2019. Doi 10.1016/j.ifacol.2019.10.018

[18] ISO. ISO 50001:2011. Sistemas de gestión de la energía - Requisitos con orientación para su uso. Leido en https://www.iso.org/obp/ui/\#iso:std:iso:50001:ed-1:v1:es

[19] Supekar, S. D., Graziano, D. J., Riddle, M. E., Nimbalkar, S. U., Das, S., Shehabi, A., \& Cresko, J. (2019). A Framework for Quantifying Energy and Productivity Bene-fits of Smart Manufacturing Technologies. Procedia CIRP, 80, 699-704. Doi: 10.1016/j.procir.2019.01.095

[20] Chacón-Ramirez, E., Zapata-Madrigal, G., \& Uribe-Hernández, J. An architecture for energy management in SMEs. Reporte Técnico de SIMAC. Enviada para su evaluación.

[21] Mohamed, N., Al-Jaroodi, J., \& Lazarova-Molnar, S. (2019). Leveraging the capabili-ties of 
industry 4.0 for improving energy efficiency in smart factories. Ieee Access, 7, 18008-18020. Doi: 10.1109/ACCESS.2019.2897045

[22] Faller, C., \& Feldmüller, D. (2015). Indus-try 4.0 learning factory for regional SMEs. Procedia Cirp, 32, 88-91. Doi: 10.1016/j.procir.2015.02.117.

[23] Zhang, M.; Zuo, Y. \& Tao, F. (2018). Equipment energy consumption manage-ment in digital twin shop-floor: A frame-work and potential applications 2018 IEEE 15th International Conference on Network-ing, Sensing and Control (ICNSC), $1-5$ 